\title{
Grazing Molecule Excitation as a Tool to Analyse the Amino Acid Sequence in Oligopeptides
}

\author{
H. Jungclas, ${ }^{1}$ L. Schmidt, ${ }^{1}$ V. V. Komarov, ${ }^{2}$ and A. M. Popova ${ }^{2}$ \\ ${ }^{1}$ Faculty of Chemistry, Philipps University of Marburg, 35032 Marburg, Germany \\ ${ }^{2}$ Skobeltsyn Institute of Nuclear Physics, Lomonossov Moscow State University, Moscow 119991, Russia
}

Correspondence should be addressed to H. Jungclas, jungclas@staff.uni-marburg.de

Received 10 June 2011; Accepted 14 August 2011

Academic Editor: Colm T. Whelan

Copyright ( $) 2011 \mathrm{H}$. Jungclas et al. This is an open access article distributed under the Creative Commons Attribution License, which permits unrestricted use, distribution, and reproduction in any medium, provided the original work is properly cited.

\begin{abstract}
A novel mass spectrometric method to analyse the sequence of amino acid residues in oligopeptides is proposed. Amino acid residues in peptide molecules contain chain-like structures of identical $\mathrm{CH}$ dipoles (IR antennas), which acquire IR energy quanta by interaction with periodic Coulomb fields and accumulate vibration excitation energy. This can subsequently lead to the dissociation of specific trap bonds inside the peptide molecule. Such excitation and dissociation processes are assumed to occur when peptide ions graze at atomic distance along a set of screened charges on a surface. These processes of grazing molecule excitation (GME) and dissociation (GMD) were applied to analyse sequences of oligopeptides by using TOF mass spectrometry. At specific grazing velocities the experimental fragment ion spectra of oligopeptides must contain a peak of high abundance corresponding to the N-terminal amino acid. This specific property of GMD offers the possibility to determine the amino acid sequence of oligopeptides.
\end{abstract}

\section{Introduction}

The general interest in peptides has resulted in developments of novel methods to determine or confirm the amino acid sequence. Many of these methods combine matrixassisted laser desorption/ionization with time-of-flight mass spectrometry (MALDI-TOF MS) [1-5]. As pointed out in these articles, the developed methods can serve to obtain peptide sequence information, which can be ambiguous yet, if used for an unknown peptide.

A method to determine sequences of amino acid residues in oligopeptides is suggested here, which is a practical application of GMD induced by IR photons [6-9]. The basis of the method is a special vibrational excitation process, which occurs to peptide molecules irradiated by IR photons. The main condition for excitation and dissociation of the molecules is the presence of chain-like substructures in these molecules. These chains are supposed to consist of periodically located diatomic valence group dipoles like $\left(\mathrm{CH}_{2}\right)_{n}$, which can serve as antennas for external radiation in the IR frequency range.

In the presented method the periodical Coulomb field is used experienced by grazing molecules, which are sliding at a velocity below Bohr velocity along a set of four or more screened atomic charges of surface atoms [7]. The resulting excitation is equivalent to IR photon irradiation.

In our experimental device, projectile molecules are grazing parallel to the surface for approximately $100 \AA$ during a time period of about $10^{-12} \mathrm{~s}$ at a minimum distance of about $2 \AA$ from the surface. The velocity component of the grazing molecule normal to the surface is close to zero. Under these conditions only the Coulomb interaction between the grazing molecules and the surface atoms is essential. All other processes, for example, molecular scattering and charge transfer, resonant electron transfer, and Auger, are negligible. For grazing peptide ions we observed that the N-terminal fragments of amino acid ions are predominant in the spectrum of their dissociation products. This phenomenon is explained in the presented paper within the framework of our excimol excitation theory and opens the chance to develop a new peptide sequencing method based upon grazing molecule excitation (GME).

Our Coulomb field excitation model for dipoles inside grazing molecules is based firstly on the well-known quantum theory of velocity-dependent resonant excitation of dipoles passing charged particles [10] and secondly on 
the analytical evaluation of excitation probabilities using Fourier expansion for a linear set of a few periodically located screened charges (see appendix [7]).

\section{Vibrational Excitation and Energy Transport in Grazing Oligopeptide Ions}

The accumulation of vibration energy and the energy transport in grazing oligopeptide molecules are affected by their Coulomb properties and structures. Protonated oligopeptides are organic molecular ions of the type

$$
\begin{aligned}
& \mathrm{NH}_{3}{ }^{+}-\mathrm{CHR}_{1}-\mathrm{CO}-\mathrm{NH}-\mathrm{CHR}_{2}-\mathrm{CO} \cdots \\
& \mathrm{NH}-\mathrm{CHR}_{m-1}-\mathrm{CO}-\mathrm{NH}-\mathrm{CHR}_{m}-\mathrm{COOH},
\end{aligned}
$$

where $\mathrm{R}_{i}(i=1 \cdots m)$ are amino acid residues and $m$ is the number of these residues. The hydrocarbon chains $\left(\mathrm{CH}_{2}\right)_{n}$ of the amino acid residues are considered as antennas for IR radiation. In these molecular antennas collective vibration excitations (excimols) are resonantly and coherently produced and accumulated when IR radiation interacts with a frequency matching the excimol frequency.

As it was shown in [6], diatomic $\mathrm{CH}$ dipoles in the antenna can be considered as a chain of coupled quantum oscillators, each of them having two vibration states. These states are the ground state and the first vibration excited state of an isolated diatomic dipole. However, instead of the first vibration state of the dipoles, a band of collective vibration states is effective in the antenna structure. This band is a consequence of the dipole-dipole interaction between the diatomic dipoles in the antenna [6].

The lowest isolated vibration state in the band (excimol) has the energy $E_{\text {ex }}=\hbar \omega_{\text {ex }}$. Each excimol in the antenna $\left(\mathrm{CH}_{2}\right)_{n}$ has an energy $E_{\text {ex }}=0.074 \mathrm{eV}$ and a lifetime $\tau_{\mathrm{ex}}=$ $10^{-11} \mathrm{~s}$ and can be excited by radiation with a frequency $\omega_{\mathrm{ex}}=1.2 \cdot 10^{14} \mathrm{~s}^{-1}$ [7]. A number of $K_{i}$ excimols in the antenna of the residue $\mathrm{R}_{i}$ can be excited independently, but they are not accumulated in a single antenna dipole due to their unharmonicity. During the radiation time $\tau_{R}<\tau_{\text {ex }}$ each dipole in the antenna can be excited $N=\tau_{R} / \tau_{\text {tr }}$ times, where $\tau_{\mathrm{tr}}=h / E_{\mathrm{tr}}$. Here $\tau_{\mathrm{tr}}$ and $E_{\mathrm{tr}}$ are the time and the energy of a vibration excitation transition from one dipole to the next [7]. The number of $K_{i}$ excimols accumulated in an antenna of residue $\mathrm{R}_{i}$ can be defined from [9, equation (8)] by the relation

$$
K_{i}=M_{i} N P_{01}
$$

where $P_{01}=P_{\mathrm{ex}} \tau_{R}$ and $P_{\mathrm{ex}}$ is the probability to excite one excimol per time unit in the antenna. $M_{i}$ is a real number of $\mathrm{CH}$ dipoles in the antenna of $\mathrm{R}_{i}$. The function $P_{\text {ex }}$ can be calculated in the frame of the time-dependent perturbation theory by the equation

$$
P_{\mathrm{ex}}=\left(\frac{8 \pi^{2} e^{2}}{3 \hbar c E_{\mathrm{ex}}}\right) D_{0}^{2} \mathfrak{M}^{2} F
$$

where $\mathfrak{M}$ is the excimol excitation matrix element, $D_{0}$ is the dipole momentum value of the diatomic dipole, and $F$ is the IR radiation fluency. The probability function $P_{\mathrm{ex}}{ }^{\text {coul }}$ of the excimol excitation per time unit in the antennas of the grazing oligopeptides by an external periodic Coulomb field is defined by [7, equation (28)]. The periodic Coulomb field fluency was obtained in the form

$$
\begin{aligned}
F_{\text {coul }}= & \left(\frac{Z^{2} e^{2} c \omega_{\mathrm{ex}}}{2 v_{\mathrm{gr}} b^{3}}\right) \\
& \cdot \exp \left\{-2 \mathrm{R}_{z}\left[\left(a_{\mathrm{eff}}^{-1}\right)^{2}+\left(\frac{\omega_{\mathrm{ex}}}{v_{\mathrm{gr}}}\right)^{2}\right]^{1 / 2}\right\},
\end{aligned}
$$

where $a_{\text {eff }}$ is the effective radius of the screened charges $Z$ of surface atoms and $b$ is the distance between these charges.

During the grazing time multiexcimol excitation in the dipole chain of each $\mathrm{R}_{i}$ occurs independently. Consequently, in each antenna of $\mathrm{R}_{i}$ the accumulated excimol energy $E\left(K_{i}\right)$ is defined by the relation

$$
E\left(K_{i}\right)=K_{i} E_{\mathrm{ex}}=M_{i} N P_{\mathrm{ex}}{ }^{\text {coul }} E_{\mathrm{ex}} \tau_{\mathrm{gr}} .
$$

The excimol energy can hop from $\mathrm{CH}$ antenna dipoles to a trap bond dipole in the oligopeptide molecule as a radiation-less transfer. This transfer occurs independently in each residue due to the dipole-dipole interaction between antenna dipoles and a trap bond dipole. Transition of the accumulated excimol energy into a specific trap bond causes dissociation of this bond if the sum of energies transmitted to trap bond $(B)$ is equal or exceeds its binding energy $E\left(K_{i}\right) \geq E_{d}(B)$. The analytical expression for the probability function $P_{f}$ for dissociation of a trap bond was presented by $\left[9\right.$, equation (16)]. The function $P_{f}$ as a function of the accumulated excimol energy has a maximum, when the excimol energy transferred to trap bond $(B)$ has the value

$$
E\left(K_{i}\right)=E_{d}(B)+\hbar \omega_{B},
$$

where $\omega_{B}$ is the eigenfrequency of trap bond dipole $(B)$. The value of $\hbar \omega_{B}$ defines a half-width of this maximum. Combining (4), (5), and (6), one can obtain an analytical expression for the periodic Coulomb field intensity $F_{\text {coul }}$ max for which the dissociation probability of trap bond $(B)$ has a maximum

$$
F_{\text {coul }}^{\max }=\left(E_{d}+\hbar \omega_{B}\right) \cdot\left[\left(\frac{8 \pi^{2} e^{2}}{3 \hbar c}\right) M_{i}\left(\frac{\tau_{R}^{2}}{\tau_{\text {tr }}}\right) D_{0}^{2} \mathfrak{M}^{2}\right]^{-1} .
$$

From (4) and (7) one can obtain the relation

$$
\begin{aligned}
E_{d}(B)+\hbar \omega_{B}= & M_{i} N E_{\mathrm{ex}} \tau_{\mathrm{gr}}\left(\frac{8 \pi^{2} e^{2}}{3 \hbar c E_{\mathrm{ex}}}\right) \\
& \cdot D_{0}{ }^{2} \mathfrak{M}^{2}\left(Z^{2} e^{2} c \frac{\omega_{\mathrm{ex}}}{2} v_{\mathrm{gr}} b^{3}\right) \\
& \cdot \exp \left\{-2 \mathrm{R}_{z}\left[\left(a_{\mathrm{eff}}^{-1}\right)^{2}+\left(\frac{\omega_{\mathrm{ex}}}{v_{\mathrm{gr}} \max }\right)^{2}\right]^{1 / 2}\right\} .
\end{aligned}
$$


Here $v_{\mathrm{gr}}{ }^{\max }$ is the grazing velocity, for which the dissociation probability of the considered trap bond has maximum value.

The mentioned probability function $P_{f}$ depends also on the factor $L^{-6}$, where $L$ is a distance between the interacting antenna and the trap bond dipoles [9]. The energy transfer is possible if the interacting dipoles are not perpendicular to each other. Thus the energy transfer occurs from antenna dipoles in each $\mathrm{R}_{i}$ to the nearest trap bond dipole if the antenna dipoles and the trap bond dipole are not perpendicular.

As it follows from the oligopeptide properties the dipoles of the bonds $\mathrm{N}-\mathrm{C}_{i}$ and $\mathrm{C}_{i}-\mathrm{C}$ in the backbone are nearly perpendicular to the $\mathrm{CH}$ antenna dipoles in $\mathrm{R}_{i}$, except for the case $i=1$. Therefore the probability of energy transfer from a dipole antenna in $\mathrm{R}_{i}$ to the mentioned nearest diatomic dipoles in the backbone is negligible with the exception of the dipoles $\mathrm{C}_{1}-\mathrm{C}$ and $\mathrm{C}_{1}-\mathrm{N}$. Then, due to mentioned oligopeptide properties, the energy accumulated in the antenna of one residue does not transit to trap bonds of other residues.

Thus the source of the oligopeptide fragments in the considered dissociation process can be theoretically predicted. They are N-terminal fragments $\mathrm{NH}_{2}-\mathrm{CHR}_{1}$ produced by dissociation of $\mathrm{C}_{1}-\mathrm{C}$ bonds and the fragment $\mathrm{CHR}_{1}$ produced by dissociation of $\mathrm{C}_{1}-\mathrm{C}$ and $\mathrm{C}_{1}-\mathrm{N}$ bonds. The energy required for these dissociations is transferred from the nearest antenna dipole of $R_{1}$. Due to the energy accumulated in IR antennas of the residues $\mathrm{R}_{i}$, there are also fragments of $\mathrm{R}_{i}(i=1 \cdots m)$ and fragments generated by trap bond dissociations inside the residues $\mathrm{R}_{i}$. The fragment production probability in one residue does not influence dissociation processes in other residues of the amino acid sequence.

\section{GME-Induced Fragmentation of Protonated Oligopeptides}

The transfer mechanisms of excitation energy inside the grazing protonated oligopeptide ions in combination with the special peptide structure define the dissociation sites and thus the resulting fragment ions. It was analysed by mass spectrometry that the specific fragment ions of the peptide ion correspond not only to fragments of the residues $\mathrm{R}_{i}$ but also to the $\mathrm{N}$-terminal fragment $\mathrm{NH}_{2}{ }^{+}-\mathrm{CHR}_{1}$, where $\mathrm{R}_{1}$ is the residue of the first amino acid in the oligopeptide sequence. From our excimol theory it can be concluded that the abundance of this N-terminal fragment ion has a maximum value at a special grazing velocity $v_{\mathrm{gr}}=v_{\mathrm{gr}}{ }^{\max }$, which is estimated in the following.

Oligopeptides are composed of up to 20 different standard amino acids. In Table 1 we present the 20 possible $\mathrm{m} / \mathrm{z}$ values of $\mathrm{N}$-terminal fragment ions $X_{1 j}=\mathrm{NH}_{2}{ }^{+}-\mathrm{CHR}_{1 j}(j=$ $1 \cdots 20$ ). We must note that the value of $v_{\mathrm{gr}}{ }^{\max }$ depends on the binding energy of the trap bond $\mathrm{C}_{1}-\mathrm{C}$. The fragment $X_{1 j}$ can contain $\mathrm{C}-\mathrm{C}, \mathrm{C}-\mathrm{N}$ or $\mathrm{C}-\mathrm{S}$ trap bonds.

Since the binding energy of $\mathrm{C}_{1}-\mathrm{C}\left(E_{d} \approx 3 \mathrm{eV}\right)$ is different from the binding energies of other possible trap bonds in fragment $X_{1 j}$ ([11] and the references therein), it is possible to calculate intervals for the location of $v_{\mathrm{gr}}{ }^{\max }$, for which the accumulated excimol energy in the IR antenna in $X_{1 j}$ is equal to the energy, where the probability of $\mathrm{C}_{1}-\mathrm{C}$ bond fragmentation has a maximum.

Our calculations using (7) and (8) show that $v_{\mathrm{gr}}{ }^{\max }$ is varying for different residues $\mathrm{R}_{1 j}$ in fragment $X_{1 j}$ depending on the number of real $\mathrm{CH}$ dipoles in the IR antenna. For at least four $\mathrm{CH}$ dipoles in the antenna of $\mathrm{R}_{1 j}$, the optimal grazing velocity $v_{\mathrm{gr}}{ }^{\max }$ should be found in the intervals $[0.5-0.7] \cdot 10^{4} \mathrm{~m} / \mathrm{s}$ or $[1.5-1.9] \cdot 10^{4} \mathrm{~m} / \mathrm{s}$. If the antenna has only two or three $\mathrm{CH}$ dipoles, $v_{\mathrm{gr}} \max$ is estimated to be located in the intervals $[0.9-1.1] \cdot 10^{4} \mathrm{~m} / \mathrm{s}$ or $[1.4-1.6]$. $10^{4} \mathrm{~m} / \mathrm{s}$.

If the appropriate grazing velocity $v_{\mathrm{gr}} \approx v_{\mathrm{gr}}{ }^{\max }$ is used (see Table 1), the probability for $\mathrm{N}$-terminal fragment ion production is much higher than that for other fragments. Thus the dominant peak of the fragment $X_{1 j}=\mathrm{NH}_{2}-\mathrm{CHR}_{1 j}$ allows a direct and easy identification of the first $\mathrm{N}$-terminal amino acid among a background of other peaks in the fragment ion spectrum of an unknown grazing oligopeptide. Because of this effect the mass resolving power of our experiment is mostly sufficient for identification of the Nterminus.

\section{Experiments}

4.1. Grazing Molecule Excitation (GME) Time-of-Flight Mass Spectrometry (TOF MS). A modified time-of-flight mass spectrometer is applied for the presented experimental results. Ions of the oligopeptide sample are produced by a nitrogen laser pulse, and all cations are accelerated by the potential $U_{\mathrm{acc}}=8 \mathrm{kV}$. The instrument configuration is schematically shown in Figure 1. Molecular ions are selectively deflected by a pair of pulsed deflection plates, thus these cations reach a converter at an angle $\alpha=11.5^{\circ}$. The converter consists of a stretched aluminized foil and a grounded grid mounted parallel to the foil at the distance of $7 \mathrm{~mm}$. The converter surface is covered by a hydrocarbon or perfluorinated hydrocarbon layer. The screened charges of the hydrogen atoms of the hydrocarbon layer or of the fluorine atoms in the perfluorinated hydrocarbon layer are sources of a periodic Coulomb field. By choosing a positive converter potential the desired grazing condition for the primary cations is achieved for $U_{\text {con }}=U_{\text {acc }} \cos ^{2} \alpha=$ $7.65 \mathrm{keV}$. The strength of the homogeneous electric field in the space between the converter surface and the grid is about $10^{-4} \mathrm{eV} / \AA$ at the mentioned value of $U_{\text {acc }}$ and the angle $\alpha$. Due to Coulomb repulsion between the atomic charges of the peptide and atomic charges of converter molecules, the normal component of the projectile velocity comes to zero at a minimum distance about $2 \AA$ to the converter surface. Now the projectiles are grazing along the converter surface molecules for about $10^{-12} \mathrm{~s}$. During this grazing period the primary cations are excited (GME) and dissociated. Only positively charged dissociation products are reaccelerated by the converter potential and finally analyzed using the timeof-flight technique. For details see [11]. 
TABLE 1: Calculated grazing velocities $v_{\mathrm{gr}} \max _{\text {for }}$ for maximum yield of $\mathrm{a}_{1}$-fragment cations from oligopeptide cations grazing along a hydrocarbon surface. Some amino acids can produce $\mathrm{a}_{1}$-fragment cations at two different grazing velocity intervals.

\begin{tabular}{|c|c|c|c|}
\hline Amino acid (j) & $\begin{array}{c}m / z \text { of fragment } \\
\mathrm{NH}_{2}{ }^{+}-\mathrm{CHR}_{1 j}\end{array}$ & $\begin{array}{c}\text { Number of } \mathrm{CH} \text { dipoles in } \\
\text { antenna of } \mathrm{R}_{1 j}\end{array}$ & $\begin{array}{l}\text { Calculated grazing velocities } \\
v_{\mathrm{gr}}^{\max }\left[10^{4} \mathrm{~m} / \mathrm{s}\right]\end{array}$ \\
\hline Alanine (A) & 44 & 4 & $0.70 \pm 0.05$ \\
\hline Arginine (R) & 129 & 7 & $0.50 \pm 0.05$ \\
\hline Asparagine $(\mathrm{N})$ & 87 & 3 & $1.00 \pm 0.10 ; 1.50 \pm 0.10$ \\
\hline Aspartic Acid (D) & 88 & 3 & $1.00 \pm 0.10 ; 1.50 \pm 0.10$ \\
\hline Cysteine (C) & 76 & 3 & $1.00 \pm 0.10 ; 1.50 \pm 0.10$ \\
\hline Glutamic Acid (E) & 102 & 5 & $0.65 \pm 0.05$ \\
\hline Glutamine (Q) & 101 & 5 & $0.65 \pm 0.05$ \\
\hline Glycine (G) & 30 & 2 & $1.00 \pm 0.10 ; 1.60 \pm 0.10$ \\
\hline Histidine $(\mathrm{H})$ & 110 & 3 & $1.00 \pm 0.10 ; 1.50 \pm 0.10$ \\
\hline Isoleucine (I) & 86 & 8 & $1.60 \pm 0.05$ \\
\hline Leucine (L) & 86 & 8 & $1.60 \pm 0.05$ \\
\hline Lysine (K) & 101 & 9 & $1.90 \pm 0.05$ \\
\hline Methionine (M) & 104 & 5 & $0.65 \pm 0.05$ \\
\hline Phenylalanine $(\mathrm{F})$ & 120 & 3 & $1.00 \pm 0.10 ; 1.50 \pm 0.10$ \\
\hline Proline $(\mathrm{P})$ & 70 & 7 & $0.50 \pm 0.05$ \\
\hline Serine $(S)$ & 60 & 3 & $1.00 \pm 0.10 ; 1.50 \pm 0.10$ \\
\hline Threonine $(\mathrm{T})$ & 74 & 2 & $1.00 \pm 0.10 ; 1.60 \pm 0.10$ \\
\hline Tryptophan (W) & 159 & 3 & $1.00 \pm 0.10 ; 1.50 \pm 0.10$ \\
\hline Tyrosine $(\mathrm{Y})$ & 136 & 3 & $1.00 \pm 0.10 ; 1.50 \pm 0.10$ \\
\hline Valine $(\mathrm{V})$ & 72 & 6 & $0.60 \pm 0.05$ \\
\hline
\end{tabular}

Due to the divergence of the primary ions, we observe even at $U_{\text {con }}=7.65 \mathrm{keV}$ three different cases, which are displayed in Figure 1 as three ion paths a, b, and c:

(a) cations hit the surface and desorb secondary ions from the surface layer (SIMS);

(b) cations graze on the surface, collect excimols, and decay to fragments (GME);

(c) primary cations are reflected without any interaction (path c).

4.2. Analysis of Oligopeptide Sequence by GME TOF Mass Spectrometry. Using the modified mass spectrometer we analyzed the GME-induced dissociation spectra of the oligopeptides YGGFL, YGGFLK, and the YGGFLR for grazing velocities $v_{\mathrm{gr}}=1.05 \cdot 10^{4}, 0.98 \cdot 10^{4}$, and $0.95 \cdot 10^{4} \mathrm{~m} / \mathrm{s}$ correspondingly. The aim of this analysis is to prove that the production of $\mathrm{N}$-terminal fragment ions has a maximum probability for $v_{\mathrm{gr}}=v_{\mathrm{gr}}{ }^{\max }$ (see Table 1 ) and that the residue types following $\mathrm{R}_{1}$ do not change the probability of the $\mathrm{N}$ terminal fragment ion production.

The fragment ion spectra are presented in Figure 2. The source of the fragment production is the energy accumulated in IR antennas of the amino acid residues. For all considered oligopeptides the N-terminal fragment $\mathrm{NH}_{2}{ }^{+}-\mathrm{CHR}_{1}(\mathrm{Y})$ with $m / z 136$ appears with highest intensity for the proper grazing velocity (see Table 1 ). In addition,

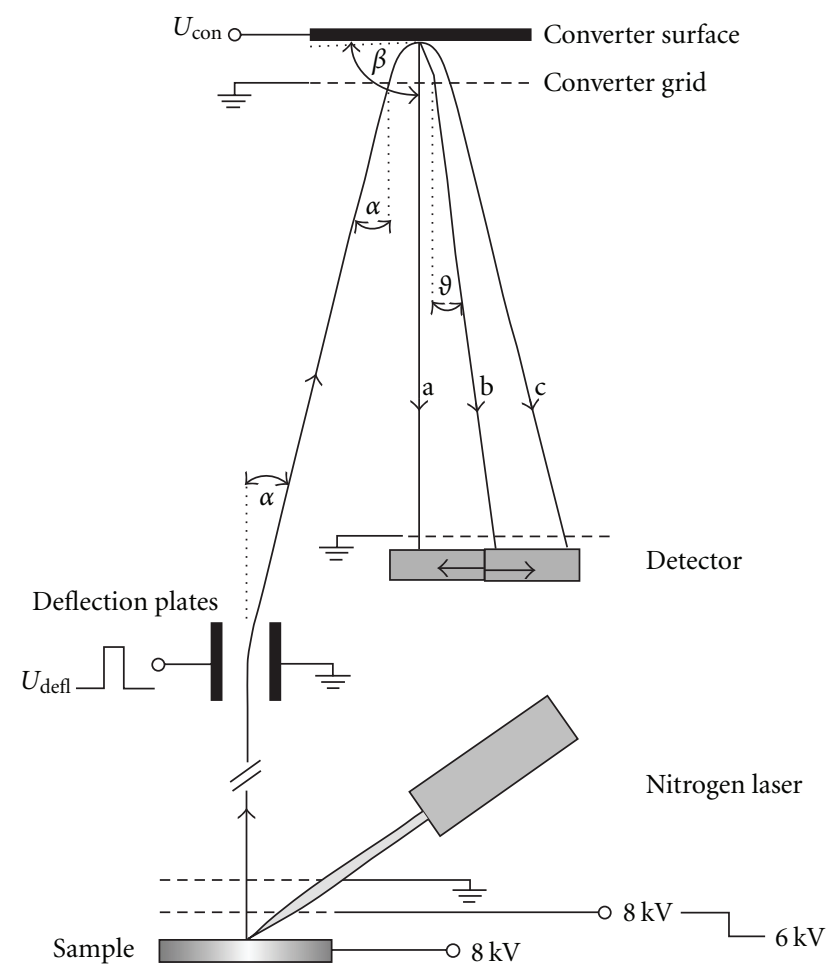

FIGURE 1: Instrumental setup for the presented experiments. In order to analyze the three ion paths $\mathrm{a}, \mathrm{b}$, and $\mathrm{c}$ the detector can be shifted (see text). 
the fragment ions $\mathrm{CHR}_{1}(\mathrm{Y})$ with $m / z 120$ and $\mathrm{R}_{1}(\mathrm{Y})$ with $\mathrm{m} / \mathrm{z} 107$ are visible in all spectra. In all spectra are also fragments of the phenylalanine residue $\mathrm{R}(\mathrm{F})$ at $\mathrm{m} / \mathrm{z} 91$ and a fragment of its dissociation at $m / z 77$, plus fragments of the leucine residue $\mathrm{R}(\mathrm{L})$ with $\mathrm{m} / z 57$ and fragments of its dissociation with $m / z 43$ and 30. In the spectrum for the oligopeptide YGGFLK there are peaks corresponding to the lysine amino acid residue $\mathrm{R}(\mathrm{K})$ with $\mathrm{m} / \mathrm{z} 73$ and fragments of its dissociation with $\mathrm{m} / z 56$ and 17 . The oligopeptide YGGFLR yields peaks corresponding to the arginine residue $\mathrm{R}(\mathrm{R})$ with $\mathrm{m} / \mathrm{z} 101$ and fragments of its dissociation with $\mathrm{m} / z 59$ and 44. Earlier [11], we measured the dissociation fragment spectrum of the grazing peptide $\mathrm{Y}(\mathrm{R})$ for $v_{\mathrm{gr}}=$ $1.4 \cdot 10^{4} \mathrm{~m} / \mathrm{s}$. Its N-terminal fragment $\mathrm{NH}_{2}{ }^{+}-\mathrm{CHR}_{1}(\mathrm{Y})$ also appears in the spectrum. Since the grazing velocity $v_{\mathrm{gr}}$ in this experiment was not matching with one of the optimal intervals (Table 1), the abundance of this fragment ion (see [11, Figure 5]) is lower than in the presented experiment. The spectra in Figure 2 show that the N-terminal fragment has a maximum abundance for $v_{\mathrm{gr}}=v_{\mathrm{gr}}{ }^{\max }$ in the interval obtained theoretically in the present work. These spectra also show that a change of amino acid residues in the sequence following the $\mathrm{N}$-terminal residue does not influence the abundance of the N-terminal fragment ion. In our previous investigations $[11,12]$ the $\mathrm{N}$-terminal fragment appears in the dissociation peptide spectra for $v_{\mathrm{gr}}$ in the interval $[0.9-1.6] 10^{4} \mathrm{~m} / \mathrm{s}$.

Using the above described feature of grazing molecule excitation (GME) it is possible to identify the first N-terminal amino acid of an oligopeptide. With the knowledge of the first amino acid, the other part of the amino acid chain can be analysed in the same way, provided a digestion method is used (e.g., in-source decay [2]), which yields enough molecules of this species. Repeating this procedure again and again, it is possible to reveal the total amino acid sequence of the oligopeptide. In each step of this procedure a suitable value of the grazing velocity has to be applied.

As described in Section 4.1 we analyze just cations. This does not mean that GME would not produce anions too.

\section{Conclusions}

In this paper we propose and experimentally demonstrate a new method to identify the first amino acid residue in oligopeptides and show how this method can be generalized for the analysis of the amino acid sequence in an oligopeptide.

The proposed sequencing method uses the grazing molecule excitation (GME) and dissociation (GMD) effect, which is able to produce cations of the N-terminal amino acid in an oligopeptide. This effect is explainable by the excimol theory worked out by us earlier [6-9]. In the frame of this theory we obtain (8), to define the grazing velocity $v_{\mathrm{gr}}{ }^{\max }$ for which the $\mathrm{N}$-terminal fragment peak in the dissociation spectrum appears with maximum probability (see Table 1).

As the dipoles $\mathrm{N}-\mathrm{C}_{i}$ and $\mathrm{C}_{i}-\mathrm{C}$ (for $i=2 \cdots m$ ) of residue $\mathrm{R}_{i}$ in the backbone of a peptide molecule are nearly

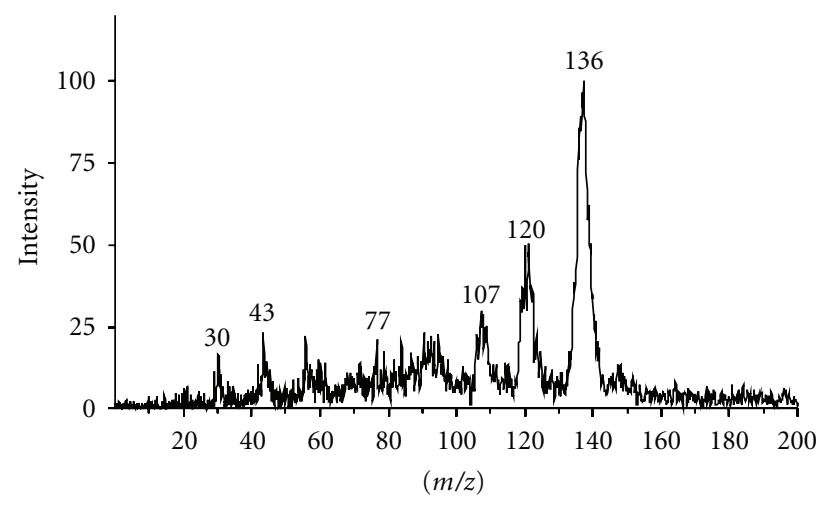

(a) YGGFL

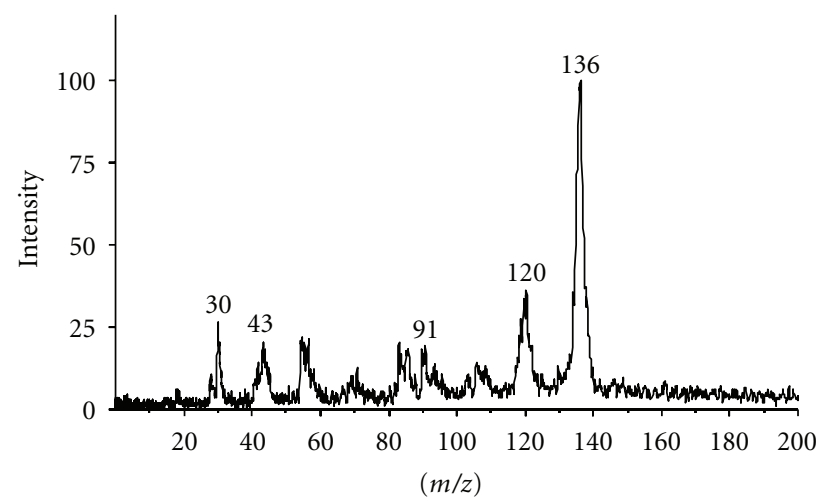

(b) YGGFLK

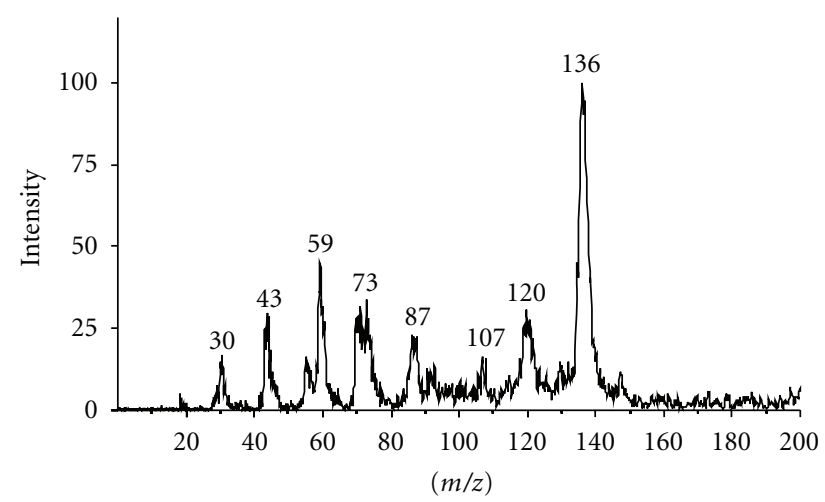

(c) YGGFLR

FIGURE 2: Mass spectra of the protonated oligopeptides YGGFL (a), YGGFLK (b), and YGGFLR (c) after GME-induced fragmentation. The peak at $m / z 136$ in each of the spectra corresponds to the ion $\mathrm{NH}_{2}{ }^{+}-\mathrm{CHR}_{1}(\mathrm{Y})$, where $\mathrm{R}_{1}(\mathrm{Y})=\mathrm{CH}_{2}-\mathrm{C}_{6} \mathrm{H}_{4}-\mathrm{OH}$ is the residue of the $\mathrm{N}$-terminal tyrosine amino acid.

perpendicular to the $\mathrm{CH}$ dipoles in $\mathrm{R}_{i}$, the probability for energy transfer from dipoles in the residue $\mathrm{R}_{i}$ (except $i=1$ ) to the nearest trap bond dipoles in the backbone and their dissociation is negligible.

We proved experimentally that the fragment production probability in one residue does not influence dissociation processes in other residues of the amino acid sequence. Amino acid residues in the sequence following the $\mathrm{N}$ terminal residue do not influence the production probability of the N-terminal fragment ion in GME. 
Thus one can find the fragment $\mathrm{NH}_{2}{ }^{+}-\mathrm{CHR}_{1}$ (fragment type $a_{1}$ ) if an appropriate grazing velocity is selected. If a sample is prepared and digested in a way [2], which produces the complete ladder, it is possible to mass select the digestion products one after the other, for example, in a time-offlight mass spectrometer, and identify the $\mathrm{N}$-terminal amino acid for each of them in the GME-induced fragmentation spectrum. This way the complete amino acid sequence of the oligopeptide can be determined.

Using our grazing molecule excitation (GME) timeof-flight (TOF) mass spectrometer, we can determine the first amino acid in a considered oligopeptide sequence. Then we discover the first amino acid in the rest part of the oligopeptide and so on up to the last amino acid in the sequence. For each step of the investigation we must successively select at least three different values for the grazing velocity $v_{\mathrm{gr}} \max \left(0.6 \cdot 10^{4} \mathrm{~m} / \mathrm{s}, 1.0 \cdot 10^{4} \mathrm{~m} / \mathrm{s}\right.$, and 1.5 . $10^{4} \mathrm{~m} / \mathrm{s}$; see Table 1) and measure the yield of $\mathrm{a}_{1}$-fragment cations for each of them.

The advantage of the presented method is the simplicity of interpretation of the measured spectra due to the appearance of pronounced $\mathrm{N}$-terminal amino acid fragment ion peaks.

\section{References}

[1] A. Pons, C. Richet, C. Robbe et al., "Sequential GC/MS analysis of sialic acids, monosaccharides, and amino acids of glycoproteins on a single sample as heptafluorobutyrate derivatives," Biochemistry, vol. 42, no. 27, pp. 8342-8353, 2003.

[2] L. A. Marzilli, T. R. Golden, R. J. Cotter, and A. S. Woods, "Peptide sequence information derived by pronase digestion and ammonium sulfate in-source decay matrix-assisted laser desorption/ionization time-of-flight mass spectrometry," Journal of the American Society for Mass Spectrometry, vol. 11, no. 11, pp. 1000-1008, 2000.

[3] R. S. Brown, B. L. Carr, and J. J. Lennon, "Factors that influence the observed fast fragmentation of peptides in matrix-assisted laser desorption," Journal of the American Society for Mass Spectrometry, vol. 7, no. 3, pp. 225-232, 1996.

[4] C. Basic, J. M. Bailey, and T. D. Lee, "An electrospray ionization study of some novel alkylamine thiohydantoin amino acid derivatives," Journal of the American Society for Mass Spectrometry, vol. 6, no. 12, pp. 1211-1220, 1995.

[5] T. Yalcin, I. G. Csizmadia, M. R. Peterson, and A. G. Harrison, "The structure and fragmentation of $\mathrm{Bn}(n \geq 3)$ ions in peptide spectra," Journal of the American Society for Mass Spectrometry, vol. 7, no. 3, pp. 233-242, 1996.

[6] V. V. Komarov, L. Schmidt, H. W. Fritsch, and H. Jungclas, "Complex dynamics variables for molecules and molecular clusters," Computational Materials Science, vol. 2, no. 3-4, pp. 427-432, 1994.

[7] H. Jungclas, A. Wieghaus, L. Schmidt, A. M. Popova, and V. V. Komarov, "Fragmentation of molecules sliding along surfaces in the speed range above thermal and below Bohr velocity," Journal of the American Society for Mass Spectrometry, vol. 10, no. 6, pp. 471-482, 1999.

[8] H. Jungclas, L. Schmidt, V. V. Komarov, A. M. Popova, and I. O. Stureiko, "Resonant IR photon induced dissociation in organic molecules with chain-like substructures," Zeitschrift fur Naturforschung —Section A, vol. 57, no. 5-6, pp. 270-276, 2002.

[9] H. Jungclas, V. V. Komarov, A. M. Popova, L. Schmidt, and I. O. Stureiko, "Hydrocarbon substructures of organic molecules as antennas and amplifiers for external mid-IR radiation," Zeitschrift fur Physikalische Chemie, vol. 221, no. 8, pp. 10751095, 2007.

[10] A. S. Dawydow, Quantenmechanik, VEB Deutscher Verlag der Wissenschaften, Berlin, Germany, 1967.

[11] A. Wieghaus, L. Schmidt, A. M. Popova, V. V. Komarov, and H. Jungcls, "Grazing incidence surface-induced dissociation of protonated peptides generated by matrix-assisted laser desorption/ionization," Rapid Communications in Mass Spectrometry, vol. 14, no. 18, pp. 1654-1661, 2000.

[12] H. Jungclas, L. Schmidt, V. V. Komarov, A. M. Popova, and I. O. Stureiko, "Local heating and dissociation of organic molecules by IR fields," Zeitschrift fur Naturforschung-Section A, vol. 59, no. 12, pp. 964-970, 2004. 

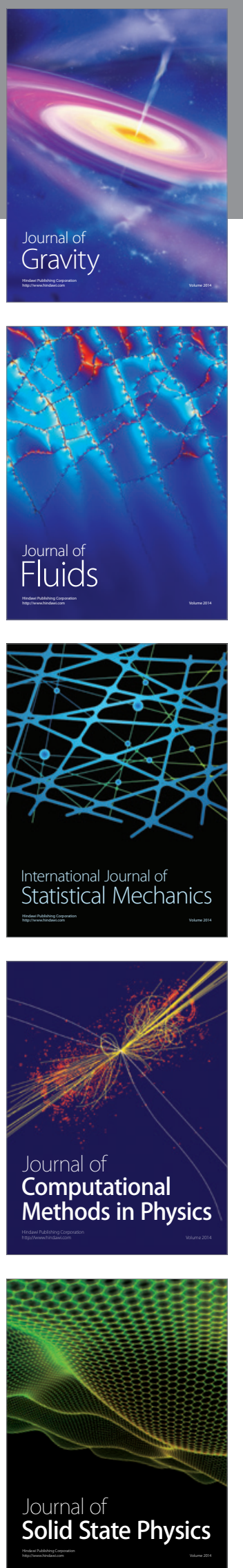

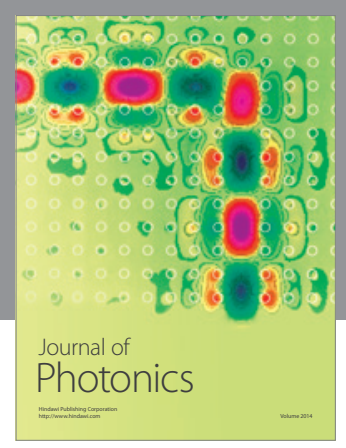

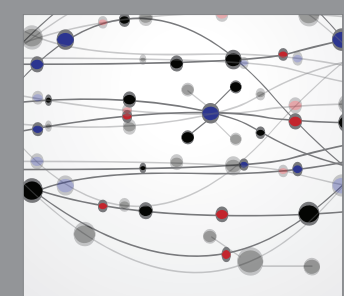

The Scientific World Journal
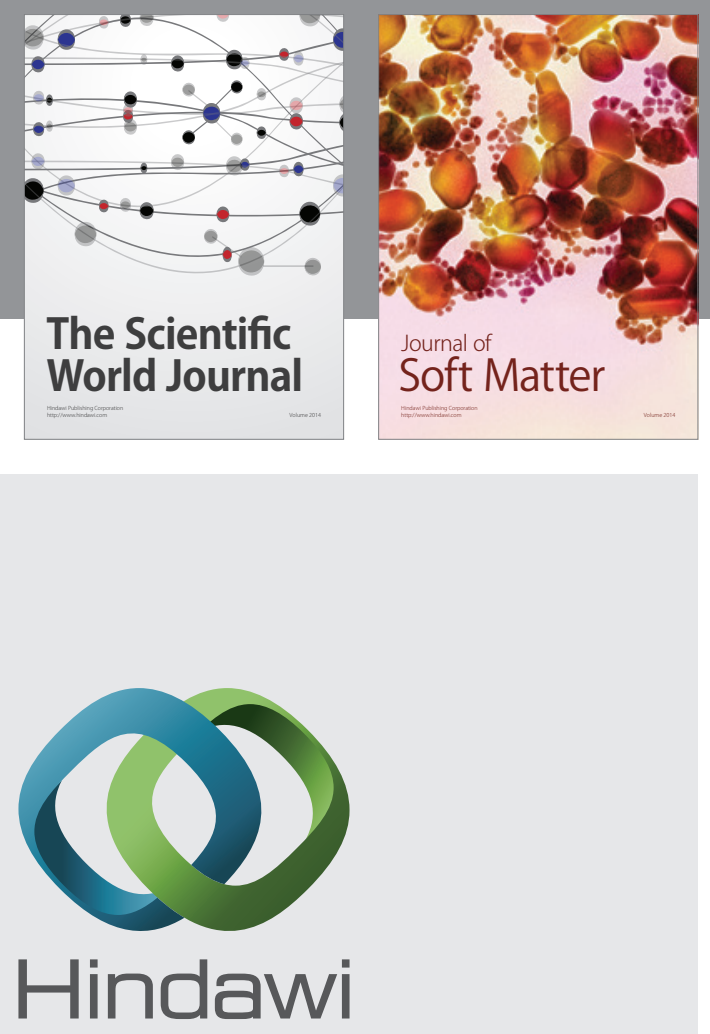

Submit your manuscripts at

http://www.hindawi.com
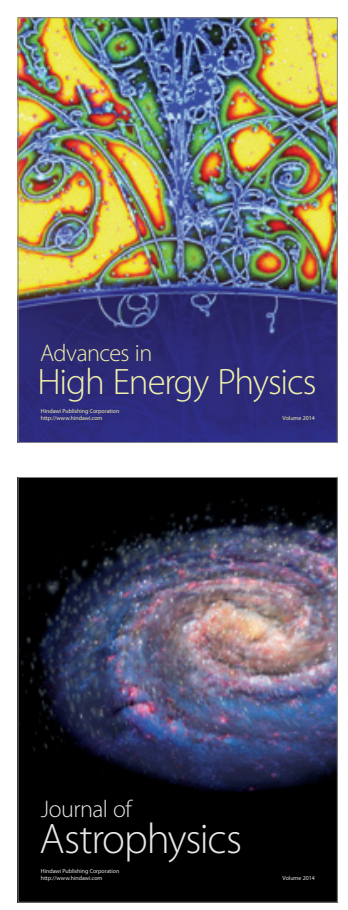
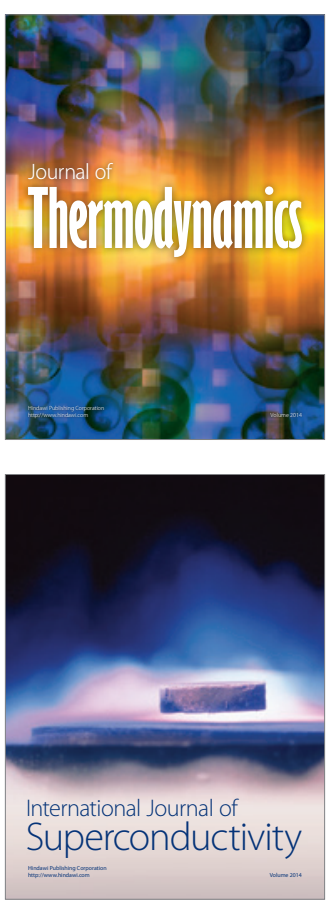
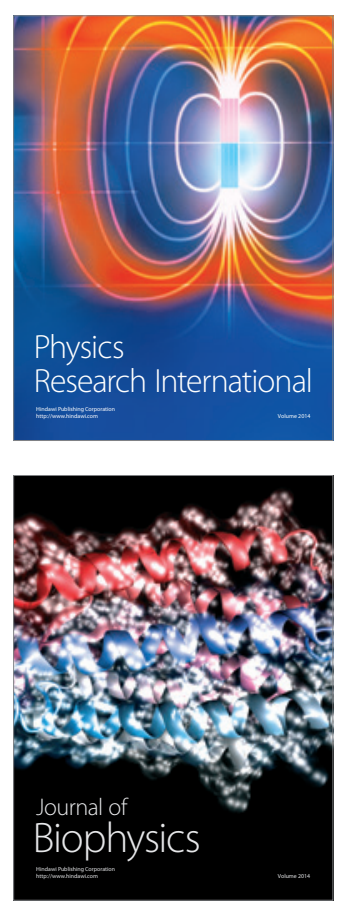
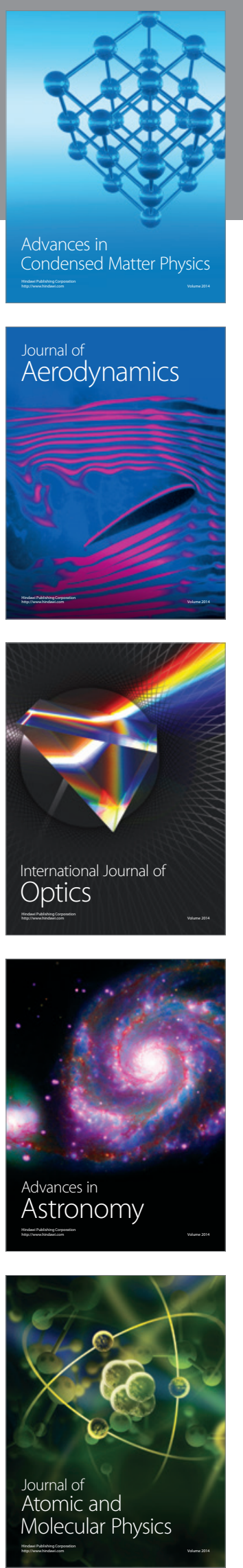